\title{
On Top of Tabletop: a Virtual Touch Panel Display
}

\author{
Li-Wei Chan, Ting-Ting Hu, Jin-Yao Lin, Yi-Ping Hung, Jane Hsu \\ Graduate Institute of Networking and Multimedia \\ Department of Computer Science and Information Engineering \\ National Taiwan University
}

\begin{abstract}
In the real world, a physical tabletop provides public and private needs for people around the table. For competing scenarios such as playing a poker game or running a price negotiation around a tabletop system, privacy protection is obviously an indispensable requirement. In this work we developed a privacy-enhanced tabletop system composed of two kinds of displays, the tabletop surface and the virtual panel. All users share the large tabletop surface as a public display while every user is provided with a virtual panel emerging above the tabletop as a personal display for viewing private information. The virtual panel is an intangible, privacy-protected virtual screen created by a special optical mechanism which offers several promising characteristics, making it perfect to be integrated into a tabletop system. The contributions of the paper include: Firstly, we introduce a novel display technique, the virtual panel, into a tabletop system to build a privacy-enhanced tabletop system. Secondly, an analysis on display optics of the virtual panel is presented to explore other potentials of the display and to claim the feasibility of the proposed combination. Thirdly a computer vision-based interaction technique is proposed to provide direct-touch interaction for the virtual panel. Finally, we discuss a wide range of considerations on designing the user interface and interaction for the virtual panel.
\end{abstract}

\section{Introduction}

The advances in display and input capabilities on computers have led to booming popularity of tabletop systems [5][13][17]. In the near future, the table will no longer be just a familiar piece of furniture at home, but a medium that affords delivering a media-rich life in our living space. A table is naturally considered as a shared workspace where people are gathered around for sharing information and collaboration. Following the observation, ex-

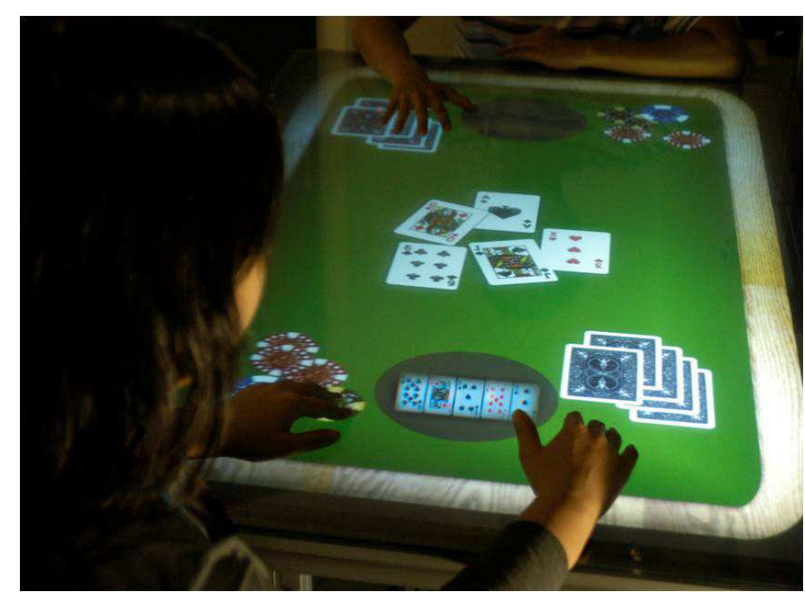

Figure 1. The users are playing poker game on the privacy-enhanced tabletop system (first prototype). Note that the image of the virtual panel is retouched for clarity.

isting research in literature draws considerable attention on facilitating computer-supported co-located collaborations, but only very few previous works have dealt with privacy problems.

In the real world, a physical tabletop provides with both public and private needs for people around the table. Central area of a tabletop is considered as a large, shared workspace where people can spread out information for sharing, while the peripheral area of the tabletop are personal areas where they can keep private information from the prying eyes of other people. In this work we developed a privacy-enhanced tabletop system composed of two kinds of displays, the tabletop surface and the virtual panel. Working with the system, all users share the large tabletop surface as a public display while every user is provided with a virtual panel as a personal display for viewing private information (see Fig.1). The virtual panel is created 
by a special optical mechanism which offers a promising characteristic, and privacy protection. The virtual panel is only visible for the user within a limited range of viewing angle so that the privacy is protected. As the nature of virtual panel is suitable for presenting privacy information, the practicability of the displaying technique is needed to be identified. In the paper, an analysis on the optical mechanism is given to investigate the practicability of introducing the mechanism into a tabletop system. In contrast to an interactive tabletop display where the users physically touch the table surface, the virtual panel on display, is intangible and floats in the air. The viewer is able to see the virtual panel, and to locate it in 3D, but is only allowed to touch it with no tactile feedback. Well-known user interfaces and interaction techniques that had been proposed for tabletop systems are requiring re-evaluation to be used for virtual panel.

\section{Related work}

Researchers have developed different approaches to deal with the needs of privacy protection under different configurations. In the following, we mainly focus on works targeting at tabletop scenario, which are highly related to the proposed system. Other works addressing distinct aspects are also included to explore the potential to be applied in a tabletop system.

One simple solution to deliver privacy information is using a separate screen as a private channel for each user. In [8], they presented an augmented tabletop game which allows the users to access private game information through the PDAs. Caretta [10] is a system for urban planning simulation that integrates PDAs and a tabletop display for personal and public spaces. In their work, the PDA is a tool for individual users to examine their ideas. Instead of carrying extra screens, i-Land [2] presented embedding the interactive components into furniture in living space. The users can make personal notes with their chairs and also interact remotely on the interactive table.

Another solution is to have each user wearing a supplemental device to provide an immersive experience. The two-user responsive Workbench [1] displays independent stereo views for two users. Single Display Privacyware [4] extends the approach to make private information visible only to the corresponding user through shutter glasses. IllusionHole [14] integrated two liquid crystal projectors and polarizing filters into a tabletop setting, which allows multiple users wearing a pair of polarized glasses to simultaneously observe stereoscopic images. Their system targeted an exhibition purpose, but can also be applied to provide individuals a private view of the displayed content. In [9], four users individually receive sound from a private audio channel while using a shared tabletop display.
For other solutions better integrated with tabletop system, the users have no need to carry on or wear extra devices. In [11], a tabletop system is built with a display mask having a hole in the center. The mask is placed over the display surface at a suitable distance. Each user observes the display through the hole to see a circular sub-region of the display. The user can move the head position to see a different part of the display, and thus to switch between public and private spaces. Their work has limitations in resolution because only a part of the display surface is used for each user. Additionally, as the visible region for each user is at a distance behind the hole, the user is only allowed to indirectly manipulate the displayed information, e.g. by a joystick. Lumisight [12] is built with Lumisty film and Fresnel lens to provide multiple views on a single tabletop. With the view-dependent property, they provide public and private spaces on the tabletop at the same time. While both support view-dependent feature, our system outperforms Lumisight by supporting spectator experience and direct touch interaction. By use of lumisity films and a Fresnel lens, the entire surface of Lumisight is privately viewable for each player, and the public display is achieved with identical content on all private displays. There is no spectator view to the (poker) game on Lumisight. In addition, Lumisight dose not support direct touch interactions. In comparison, the public display of our system inherits all good features from existing tabletop system including the support of spectator experience and direct touch. We also provide direct touch support for the private virtual panels. Wu [6] applies gesture-based solution to handle privacy problem. Their approach detects a tilted horizontal hand gesture on the tabletop and uses the hand as physical space upon which to project private information.

\section{An overview of virtual panel}

The construction of virtual panel is based on the display optics of image formation by convex lens. As the optics is quite simple, it has potential to create a new kind of display technique. The applied display optics has several limitations due to the principles of optics. However the limitations can also be the unique features to meet some specific needs of applications. In the following, we first introduce the basic principles of the display optics and then mention several considerations when deploying the optics in distinct applications.

\subsection{The Display Optics}

A convex lens, also named a positive or converging lens, is used as an optic component which concentrates beams to form a real image in air. A parallel beam of light travelling perpendicular to the lens would converge to a spot on the 


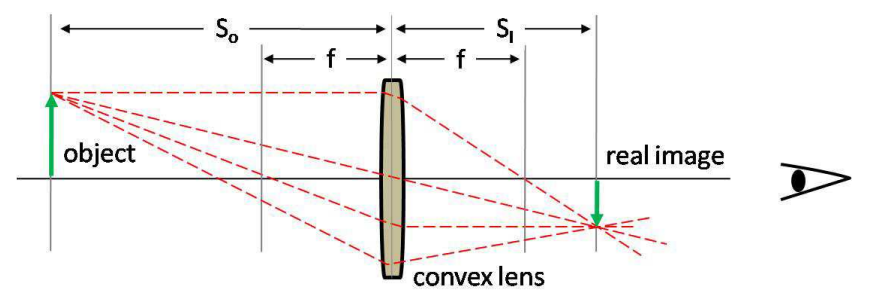

Figure 2. This figure illustrates the basic image formation by convex lens. If an object is placed at a distance larger than $f$ along the axis in front of the lens, an image of the object will be formed at a distance behind the lens.

axis, at a distance $\mathrm{f}$, the focal length of the lens, behind the lens. As shown in Fig.2, an object at a distance larger than $\mathrm{f}$ along the axis in front of the lens will form an image of the object behind the lens. The distances from the object to the lens and from the lens to the image as $S_{O}$ and $S_{I}$ respectively. The thin lens formula is known as: $\frac{1}{S_{O}}+\frac{1}{S_{I}}=$ $\frac{1}{f}$. The image at $S_{I}$ is known as a real image. If we place a LCD screen at $S_{O}$, the image on the LCD screen will appear at $S_{I}$ as a virtual panel.

In the implementation, the convex lens is replaced with a Fresnel lens. Fresnel lens enables the construction of large aperture and short focal length lenses without the weight and volume of material. Compared to conventional lenses, Fresnel lens is much thinner, thus passing more light, leading to forming clearer images. By manipulating distances $S_{O}$ and $S_{I}$, and substituting lenses with various focal lengths, we can create a virtual panel appearing at different positions and sizes. However, without knowing characteristics of the optics, the formed image could be in low quality or the applied mechanism can be bulky.

\subsection{Considerations on Deployment}

Use of the optical mechanism in an interactive display is first proposed by Ikeda in [3]. They applied the mechanism to bring the image on the LCD screen into a glass ball. In this work, we apply the mechanism as a virtual panel for building a privacy-enhanced tabletop system, in which the applied parameters of the mechanism are quite different from those used in [3]. To provide more insights for designers, we give an analysis on the optical mechanism. In the following, some factors that need to be taken into consideration are listed.

\subsubsection{Decision of Distance $S_{O}$}

According to the thin lens formula, moving the LCD screen to a distance $S_{O}$ will transport the screen image at a position $S_{I}$, with corresponding magnification of the image on the other side of the lens. The magnification can be calculated as $\frac{S_{I}}{S_{O}}$. However, it is not the case as the transported image can appear clearly at any desired position $S_{I}$ by manipulating $S_{O}$ arbitrarily, which highly depends on the application expected. To produce a real image, $S_{O}$ has to be at least larger than focal length length $f$.

Optical Reduction $\left(S_{O}>2 f\right)$ : In this case, the formed image is smaller than the LCD screen according to the formula. As the formed image is optically reduced and while still retaining the resolution of the LCD screen, the formed image will be more sophisticated. However, if an application is willing to produce a formed image with reasonable size, a large size of LCD screen is required. As a result, the accomplished mechanism could be bulky. With this limitation, this kind of setup is preferred to applications for exhibitions. In one of our previous work, we made a magic crystal ball [16] based on the setup, in which the Fresnel lens having 8.2 inches in focal length is used. We place a 17 -inches LCD screen at $S_{O}$ equal to $3 f$, which produced a 12-inches image penetrating into a transparent sphere. The user is allowed to see a virtual object/scene appearing inside the transparent sphere, and is able to manipulate the displayed content with bare hands. Although the optics of magic crystal ball was carefully designed, the resultant volume of the ball system is still large. However the presenting imaging is quite promising which is the key consideration for an exhibition system.

Optical Magnification $\left(f<S_{O}<2 f\right)$ : With the setup, the formed image is optically magnified. In acquisition of a desired image size, a smaller LCD screen is enough. The distance $S_{O}$ is also smaller, limited between $f$ and $2 f$, in comparison to previous setup. The volume of the resultant mechanism can be greatly reduced, and thus be more practical to be embedded in furniture in the living space. In addition, a smaller $S_{O}$ relates to a much larger $S_{I}$, which suggests the formed image appearing at a relatively large distance to the lens, making it more flexible to the needs of applications. However, use of the setup also comes with some defects that harm the quality of the perceived image. Firstly, given the size of the formed image, the resolution is limited by a relatively small LCD screen. Secondly, since the formed image is optically magnified, the brightness of the image will be diminished. In this work, the magnification setup is applied to create virtual panels. An ultra high bright LCD screen is used to compensate for brightness loss. 


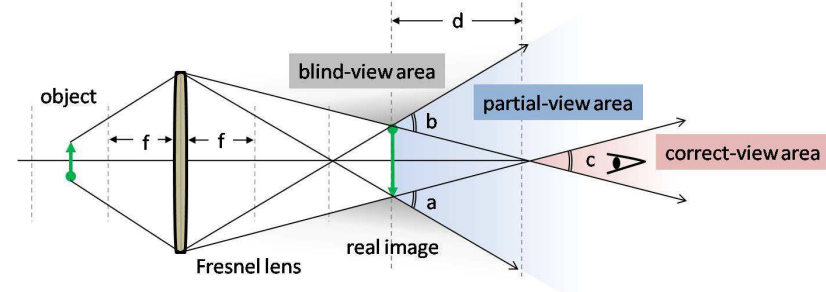

Figure 3. The viewer is required to stay in correct-view area to perceive a correct image on virtual panel. Out of the area, the viewer can only perceive a partial view or blind view of the image.
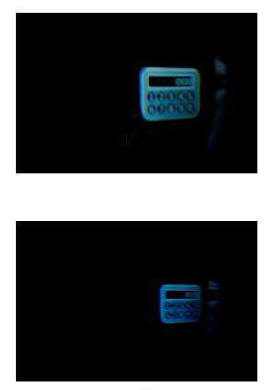

$-45^{\circ}$

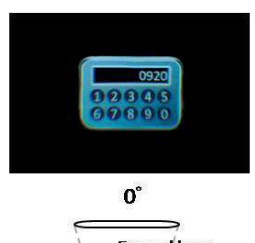

Fresnel len

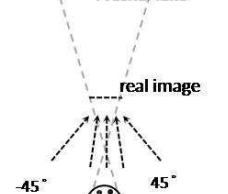

(0)

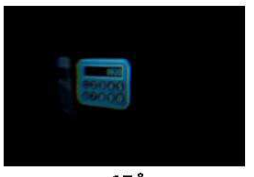

15

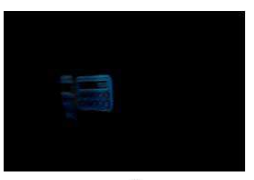

$45^{\circ}$
Figure 4. The viewer observes a password input panel displayed on the virtual panel in $45,-15,0,15,45$ degrees to the correct viewing angle.

\subsubsection{Decision of Focal Length}

The focal length of the Fresnel length relates to both $S_{O}$ and $S_{I}$, and thus influences the formed image. In acquisition of a desired image size, one can use a small/large focal length lens to obtain magnified/reduced images. However, the focal length also relates to the size of the lens, which can be a major concern in designing phase. A Fresnel lens with long focal length comes with big surface of the lens [19] and thus requires more space for installation. Noted that, as the optical magnification brings a larger image, the size of the formed image can never be bigger than the size of the Fresnel lens surface, or else, only the part covered by the lens surface can be observed.

\subsubsection{To Touch or Not To Touch}

When applying the display optics in applications, the designers need to consider whether they expect the user to touch the formed image or not in the early design phase.
With different configurations of the display optics, the formed image is able to be seen by the viewers, but is too distant to be touched by the viewers with their hands. In Fig.3, we explain the consideration with an optical magnification configuration. The object is an arrow with triangle and circle ends, positioning at one and half focal lengths on the left side of the lens in the figure. According to the formula, the formed image will be appearing at threefolds focal length with double magnification on the other side. To observe the triangle end of the formed image correctly, the viewer has to stay in the area included by the angle labeled $a$, towards the lens. Likewise, to stay in the area included by the angle $b$ to observe the circle end. As a result, to perceive the formed arrow correctly, the viewer is required to at least stay in the intersection of the two areas, named correct-view area, included by angle $\mathrm{c}$ in the figure. The correct-view area indicates the valid range of viewing angle and the valid positions to perceive a correct formed image. Staying out of the area, the viewer can only perceive a partial-view or nothing of the image. Notice that the correct-view area is positioned at a distance $\mathrm{d}$ behind the formed image. If the distance $d$ is larger than the length of the viewers hands, the viewer will not be able to touch the image.

\subsubsection{Privacy Is a Concern}

The valid viewing angle of the virtual panel is limited in a given range. Only staying in correct-view area, the viewer can see the displayed content on the virtual panel clearly and correctly. If privacy is a major concern, the designer can configure the optical mechanism so as to have the correctview area right in front of the viewers eye. Therefore, other viewers behind or next to the target viewer can only observe a distorted, partial, or blind view of the displayed content. In Fig.4, we show the viewer observes a password input panel displayed on the virtual panel in different viewing angles. The password typed by the target viewer is thus protected from any intrusion by others. On the contrary, the designer can also develop a wide range of viewing angle to have more than one viewer simultaneously see the displayed content, as in an exhibition scenario. In general, applying optical reduction, the designer can obtain more sophisticated image and flexibility in wider viewing angle $c$ and shorter touchable distance $d$, with which multipleuser interaction is possible, but requiring space for could-be bulky mechanism in order to obtain a nice viewing size. For optic magnification, a limited viewing angle can be the benefit for privacy protection, and the resultant mechanism can be relatively small, and be able to be embedded into our living space. Although virtual panel provides different levels of protection according to the viewing angle, the designer should know that the distortion from the lens is better at hiding detailed textures such as text. Color and shape can 


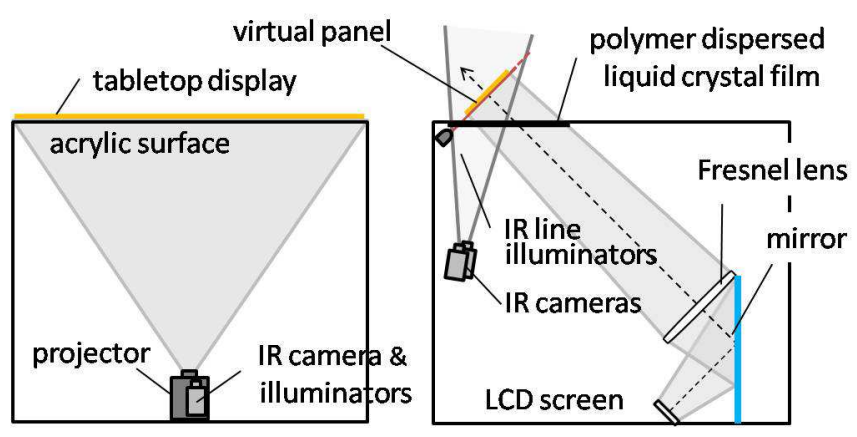

(a) Tabletop display

(b) Virtual panel

Figure 5. Two main components of the proposed tabletop system. The architecture of (a) the tabletop surface and (b) the virtual panel.

still be observed even with a large distortion.

\section{System Implementation}

The privacy-enhanced tabletop system comprises two kinds of displays, the tabletop surface and the virtual panel. In particular, the virtual panel is created by a special optical mechanism to provide the capability of privacy protection. In this section, the details of the hardware configuration that combines the two displays into a tabletop system is given. After that, the software implementation including system calibration and detection technique is provided.

\subsection{Hardware Configuration}

The architecture for the proposed tabletop system is shown in Fig.6, which consists of two main parts, the tabletop display and the virtual panel.

\subsubsection{Tabletop Display}

For the part of the tabletop display, the configuration is shown in Fig.5(a). The tabletop is made of a translucent acrylic surface. A projector is installed underneath the tabletop, bringing to a rear projection tabletop display. In order to provide interaction for the tabletop display, we set an infra-red camera and several infra-red illuminators near the projector for multi-touch detection.

\subsubsection{Virtual Panel}

To provide a virtual panel float above the tabletop, as shown in Fig.5(b), the image displayed on the LCD screen is first reflected by a mirror and then passes through the Fresnel lens (the focal length is 8.2 inches), forming a real image towards the tabletop surface. In order that the virtual panel would not be blocked by the tabletop, in the first prototype we used a transparent acrylic surface attached with tracing papers as the tabletop surface for rear-projection. A hole on the tracing paper is reserved for each virtual panel. The setup is simple and a seamless integration is achieved but the black holes are inevitable even when virtual panels are inactive. In the second prototype, the tabletop is replaced with translucent surface. We embed a polymer dispersed liquid crystal (PDLC) glass on the tabletop. PDLC consists of light changing liquid crystals capable of adjusting the transparency of the glass with external electric field provided. The region of the glass on the tabletop can be regarded as a gate for the virtual panel. As a result, the virtual panel is able to penetrate the glass and appears above the tabletop in a transparent mode. Otherwise, the glass is translucent and the whole tabletop is retained for rear projection. Because the glass has surrounding frame for electrical wires, there would be a physical thin border on the joint of the glass and the tabletop surface. It is also possible to use a PDLC film[20] to achieve advantages of the two prototypes. In the implementation, the PDLC glass is powered through a relay controlled by our computer program. When glass is in transparent mode, the projector would project black pixels on the corresponding regions of PDLC glass of the tabletop to avoid interfering with the perception of virtual panels of users and to avoid striking the fingertips and the ceiling of the room. When the glass is in diffuse mode, the different levels of diffusion between PDLC glass and the tabletop surface could cause different illumination levels from rear projection. The projector would adjust illumination level on particular regions of the glass in order to provide a homogeneous projection surface.

As the virtual panel is formed by a real image in the air, the user can locate its position in $3 \mathrm{D}$, but touching the virtual panel is simply touching the air. For interaction, we would like to know whether the user touches the panel and the contacted positions on the panel. Instead of tracking the users fingertips in 3D all the time, we setup an IR line illuminator which creates an IR plane aligned with the virtual panel. The IR line illuminator is made by attaching a cylindrical lens in front of an IR LED. The cylindrical lens stretches IR beam into a line, which intersects the space into an IR plane. When the users touch the virtual panel, they touch the IR plane as well. The IR reflection caused by the users fingertips is then identified by using IR cameras. The applied method is quite simple and effective. However, the setup of the IR line illuminators and the IR cameras is highly related to the detection performance.

Fig.7 shows a front view and a side view of the tabletop system. The dashed square indicates the virtual panel which floats above the tabletop. In the implementation, we 


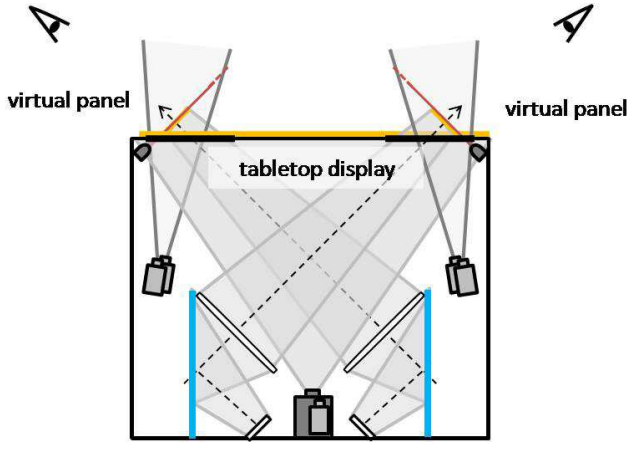

Figure 6. The architecture of the privacyenhanced tabletop system.

attached two IR-line illuminators which emit from two sides of the virtual panel. Two IR cameras are installed under the illuminators to observe the illuminations and also as large coverage of the virtual panel as possible. Assuming that the user casually operates with the virtual panel, the detection can be broken when the lower part of the palm unconsciously touches the panel and thus is unexpectedly illuminated as shown in Fig.7(b). The unexpected illumination can be removed on the detection step based on the observation described below. Fingertip is much smaller than the lower part of palm and therefore the illuminations on the fingertips on the two sides are usually shared by some intersections on the bottom. In contrast, the illuminations on two sides of the lower part of the palm usually have little or no intersection, due to the bottom being hardly be lightened by either illuminator. Based on the observation, we first transform two IR camera views to virtual panel coordinate by multiplying with corresponding homography matrix. After that we can analyze the intersection patterns of the two views to recognize the fingertips. Finding fingertips from the intersections also help improve the accuracy of fingertip positioning.

\subsection{Software Implementation}

The detection of the proposed tabletop system is to provide finger-touch interaction for both the tabletop display and the virtual panel. In the following, the construction of a multi-touch tabletop display is first described. Next, we introduce the proposed algorithm for the virtual panel including the calibration and detection steps.

\subsubsection{Finger-Touch Detection for Tabletop Display}

To provide finger-touch interaction for the tabletop display, the fingertip finding algorithm proposed in our previous work is applied. The algorithm works on images captured

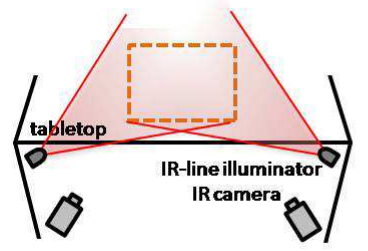

(a) front view

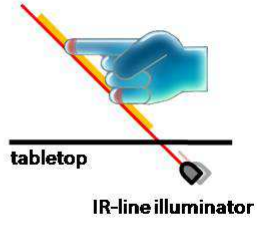

(b) side view
Figure 7. Use of Two IR-line Illuminators.

by infra-red cameras installed underneath a translucent diffusion tabletop surface looking at human gestures. On users hands approaching the tabletop lighted by the infra-red illuminators, the camera recognizes fingertips from observed infra-red reflection to provide multi-touch interaction. More details of the algorithm can be found in [15].

\subsubsection{Calibration of Virtual Panel}

The detection module for the virtual panel comprises two infrared cameras and two IR-line illuminators. As there are multiple IR-line illuminators for each virtual panel, the alignment of the virtual panel and the IR illumination plane needs to be confirmed in the first place. Once the alignment is accomplished, we compute the coordinate mappings among the two cameras and the virtual panel by finding the homography relationships.

\subsubsection{Finger-Touch Detection for Virtual Panel}

For each virtual panel, two IR cameras are used to achieve the fingertip-positioning. As the IR camera is insensitive to lighting interference in typical office environments, we can easily identify the IR reflection on the fingertips by applying background subtraction operations. Once the foreground regions on the two camera frames are identified, we transform the foreground regions onto the image plane of the virtual panel by applying homography transformation. We then extract the intersections of the foreground regions found on the two camera frames as fingertip positions. We apply finger detection method which includes connected component, rejection of false alarm by size checking, and then apply Kalman Filter for tracking fingers.

Our detection for direct-touch virtual panel is similar to the one in [7]. Both apply the stereo properties (binocular disparity) of two calibrated cameras to determine if a given object is located on a particular plane. A minor difference is that we use two IR scans to create an invisible detection surface aligning to the virtual panel while the TouchLight used an IR illuminator to directly illuminate the users hands. In our approach, using two IR-scans can avoid occlusion problems. While fingertip positions can be determined using a 


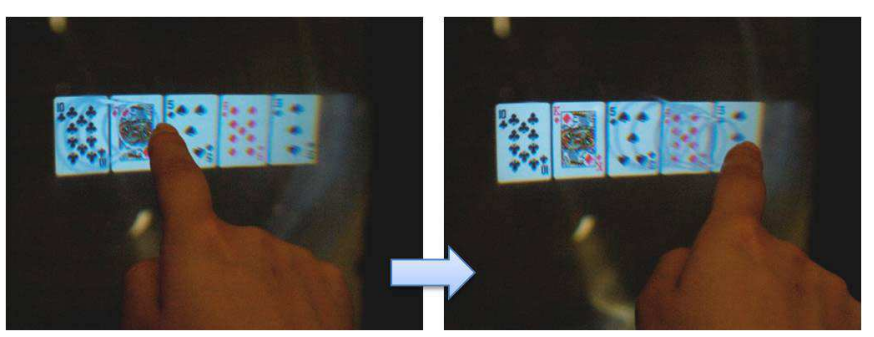

(a)

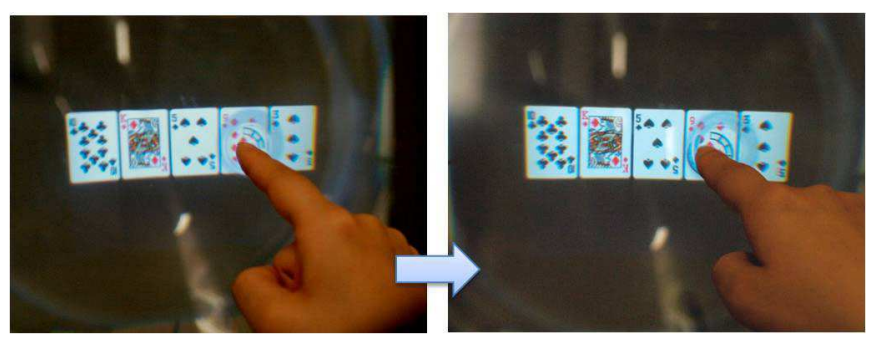

(b)

Figure 8. User interactions. (a) The ripples are generated along with the fingertip positions. (b) The waiting circle starts to expand its fans around the finger.

single camera in our IR-scan setup, we use two cameras and stereo property to further improve the detection accuracy and stability.

\section{Interaction Design}

\subsection{Virtual Plane As Water Surface}

To interact with the virtual panel, we use a water ripple metaphor to personify the virtual interaction plane. When the user contacts the virtual plane, the system will generate visual ripples on the touching points, just as the user touches water in the real world, as shown in Fig.8(a). Through the experiences we have had with water, both of tactile and visual sensations, its easy to understand the implications of this metaphor, consciously or unconsciously. Users can obtain enough feedback, to establish a link between actions, changes of interface appearance and functionalities, and further identify the affordance of the system.

The sense of touch is essential for humans to estimate object properties. In virtual panel, the intangible trait triggers another objective for designing visual interaction - to generate pseudo-haptics feedback response to direct touch. Recall the sensations we have while being in direct contact with real water; the visual phenomenon of water ripple is strongly perceived compared to the tactile sense. In contrast, contacting a solid object will result in more intensive haptic sensation than vision. Using a liquid metaphor is well suited to an intangible display since it produces feedbacks very close to nature. Furthermore, by the soft imagery of water, users are more willing to touch the virtual plane. The water, either in real or virtual world, causes no harm to humans even if someone touches it unintentionally.

\subsection{Intangible Interaction}

Simple Manipulations: The objective in this work is to integrate virtual panel with the existing tabletop systems, as a complementary display to eke out insufficiency of privacy protection. In most scenarios, the user only requires a small private region and applies simple manipulations on it. Due to the lack of sense of touch, it might cause problems for the users on precise positioning. Leveraging the users goals and skills under a private virtual display, affording simple manipulations is sufficient to the users.

Selection with Dwell Time: Selection is a required input operation in most applications. As virtual panel is a virtual display screen in the air, the users usually have a slight difference in interpreting the physical depth of the panel to the users eye position. Due to the mis-interpretation of the depth, the user has to be very careful to issue a click operation on virtual panel without frustrations. Instead of clicking directly on the virtual panel, our design encourages the user to touch the virtual panel, staying their fingers on the panel to obtain continuous visual ripple feedbacks, and then to activate a selection. To perform a selection, the user simply slides the finger to the target object, and stays for a dwell time to ensure the operation to be applied. We take an approach similar to Sony EyeToy[18]. As shown in Fig.8(b), when a user moves his finger to some object, a circle under the fingertip appears and starts to expand its fans in a specified dwell time. The selection operation will be applied after the circle is completed.

Audio Feedbacks: In virtual panel, we use non-speech audio cues to provide instant feedback about user actions and notification of system's state changes including ripple generations and selection. Audio feedbacks also help users more casually interact with the virtual panel. When sliding fingers on the water surface, users might unconsciously lower their fingers, and finally fail the fingertip detection. The audio feedbacks can help users to be aware of the depth of their fingers on the virtual panel. If the user slides fingers lightly on the virtual panel, the system responds with bright tone of ripple generations, making the users aware that they are interacting with the virtual panel correctly. Otherwise, the system responds with dark tone of ripple generations to notify the users to lifting their fingers up, when many large foreground regions are found in detection process. 


\section{Conclusion}

This paper presents a privacy-enhanced tabletop system by integrating virtual panels. Interactive tabletop is one of the most attractive human-computer interaction systems in recent years. We have designed a privacy-enhanced tabletop system comprising of two kinds of displays, the tabletop surface and the virtual panels. The virtual panel is an intangible, privacy-protected virtual screen created by a special optical mechanism. In this paper, we explored the applicability and feasibility of the virtual panel including an analysis on its display optics and several considerations on design of user interface and user interaction for virtual panel.

For future work, current realization of virtual panels has limitations needed to be addressed. The cost to build a virtual panel is high. The locations of virtual panels on the tabletop are pre-determined, which provides a physical constraint on the number and actual location of multiple users. Some other solutions to privacy-protection can be considered or integrated for this issue. For interaction, current implementation of virtual panel only provides finger-based interaction. Other interactions accommodating the intangible characteristic of virtual panel are needed to be further investigated. For example, more gestures can be defined for manipulating poker cards on virtual panels and object transitions between virtual panels and tabletop surface.

\section{References}

[1] Agrawala, M., Beers, A.C., FrLohlich, B., and Hanrahan, P. (1997). The two-user responsive workbench: support for collaboration through individual views of a shared space. ACM SIGGRAPH, p. 327-332.

[2] Streitz, N., et al. (1999). i-LAND: an interactive landscape for creativity and innovation. ACM CHI, p. 120127.

[3] Ikeda, H., Naemura, T., Harashima, H., and Ishikawa, J. (2001). i-ball: Interactive information display like a crystal ball. Abstract and Applications of SIGGRAPH, p. 122 .

[4] Shoemaker, G.B.D., and Inkpen, K.M. (2001). Single display privacyware: augmenting public displays with private information. ACM CHI, p. 522-529.

[5] Dietz, P.H., and Leigh, D.L. (2001). DiamondTouch: A multi-user touch technology. ACM UIST, p. 219-226.

[6] Wu, M., and Balakrishnan, R. (2003). Multi-finger and whole hand gestural interaction techniques for multiuser tabletop displays. ACM UIST, p. 193-202.
[7] Wilson, A. (2004). TouchLight: An Imaging Touch Screen and Display for Gesture-Based Interaction. International Conference on Multimodal Interfaces, p. 69-76.

[8] Magerkurth, C., Memisoglu, M., Engelke, T., and Streitz, N.A. (2004). Towards the next generation of tabletop gaming experiences. Proceedings of Graphics Interface, p. 73-80.

[9] Morris, M.R., Morris, D., and Winograd, T. (2004). Individual audio channels with single display groupware: effects on ommunication and task strategy. ACM CSCW, p. 242-251.

[10] Sugimoto, M., Hosoi, K., and Hashizume, H. (2004). Caretta: system for supporting face-to-face collaboration by integrating personal and shared spaces $A C M$ CHI, p. 41-48.

[11] Kitamura, Y., Osawa, W., Yamaguchi, T., Takemura, H., and Kishino, F. (2005). A display table for strategic collaboration preserving private and public information. International Conference on Entertainment Computing, p. 167-179.

[12] Kakehi, T., Iida, M., Naemura, T., Shirai, Y., and Matsushita, M. (2005). Lumisight table: an interactive view-dependent tabletop display. IEEE Computer Graphics and Applications, January/February, 25(1), p. 48-53.

[13] Han, J.Y. (2005) Low-cost multi-touch sensing through frustrated total internal reflection. ACM UIST, p. 115-118.

[14] Kitamura, Y., Nakayama, T., Nakashima, T., and Yamamoto, S. (2006). The illusionhole with polarization filters. ACM VRST, p. 244-251.

[15] Chan, L.W., Chuang, Y.F., Chia, Y.W., Hung, Y.P. and Jane Hsu. (2007). A new method for multi-finger detection using a regular diffuser. International Conference on Human-Computer Interaction.

[16] Chan, L.W., Chuang, Y.F., Yu, M.C., Chao, Y.L., Lee, M.S., Hung, Y.P. and Jane Hsu. (2007) Gesture-based interaction for a magic crystal ball. ACM VRST, p. 157164.

[17] Microsoft Surface: http://www.surface.com

[18] Sony EyeToy: http://www.eyetoy.com/

[19] Fresnel Technologies, Inc.: http://www.fresneltech.com/index.html

[20] 3G Privacy Film: http://www.scienstry.us/products.htm 